\title{
Serial anticipation and pattern extrapolation in rats as a function of element discriminability
}

\author{
STEVEN J. HAGGBLOOM and DAWN M. BROOKS \\ Arkansas State University, State University, Arkansas
}

\begin{abstract}
In Experiment 1, three groups of rats were trained on one of three serial patterns consisting of different numbers of .045- $\mathrm{g}$ food pellets, either the simple strong monotonic pattern 14-7-3-1-0 (Group SM) or one of two complex weak monotonic patterns, 14-5-5-1-0 (Group 5-5) or 14-9-1-1-0 (Group 1-1). Learning to anticipate the terminal 0-pellet element occurred faster in Group 1-1 than in Group SM, which in turn learned faster than Group 5-5. In Experiment 2, Groups SM, 5-5, and 1-1 were trained on the first four elements of the patterns experienced by their Experiment 1 counterparts and then were tested for their ability to extrapolate the series to include the addition of a 0-pellet element in the fifth position. Extrapolation behavior was better in Group 1-1 than in Group SM, which in turn performed better than Group 5-5. The results were shown to be consistent with a memory-discrimination model of serial learning and inconsistent with a rule-learning model based on pattern complexity.
\end{abstract}

Rats can learn to anticipate one or more elements of an ordered series of different numbers of .045-g food pellets. What is theoretically interesting is that some series are easier to learn than others. For example, Hulse and Dorsky (1977) reported faster learning of slow running in anticipation of nonreward, or 0 pellets, when that event terminated the strong monotonic (SM) series 14-7-3-1-0 than when it terminated the nonmonotonic (NM) series 14-1-3-7-0 or the weak monotonic (WM) series 14-5-51-0. Capaldi and Molina (1979), on the other hand, reported faster learning to anticipate 0 pellets in the NM pattern 1-29-0 than in the SM pattern 20-10-0, and better learning on the WM patterns 14-14-2-0 and 15-15-0-0 than on the SM pattern 15-10-5-0.

Hulse (1978) hypothesized that rats induce a rule governing the formal structure of a pattern, the ease of anticipation learning being directly related to the simplicity of the rule. According to this view, the SM pattern can be described by the relatively simple rule that each element (E) is smaller than the preceding element, that is, $\mathrm{E}(\mathrm{i})>$ $E(i+1)$. The WM pattern 14-5-5-1-0, on the other hand, is defined by the rule(s) $\mathrm{E}(\mathrm{i})>\mathrm{E}(\mathrm{i}+1)$ and $\mathrm{E}(2)=\mathrm{E}(3)$ (Hulse \& Dorsky, 1977). Discovering the rule $\mathrm{E}(\mathrm{i})>$ $\mathrm{E}(\mathrm{i}+1)$ is presumably easier than learning both that rule and the exception $\mathrm{E}(2)=\mathrm{E}(3)$, and so the 14-7-3-1-0 series is learned faster than the 14-5-5-1-0 series.

Capaldi (e.g., Capaldi \& Molina, 1979; Capaldi, Verry, \& Davidson, 1980a) has proposed a memory-discrimination learning model of serial learning in which the anticipatory mechanism is the signal value associated with the memory of one or more prior elements. Of very con-

These experiments were supported by a Faculty Research Grant to the first author from Arkansas State University. Requests for reprints should be addressed to the first author, Department of Psychology, Arkansas State University, P.O. Box 2127, State University, AR 72467. siderable importance to this model for learning to anticipate, say, a terminal 0-pellet element is the discriminability of the element(s) signaling 0 pellets from the element(s) signaling other, especially large, quantities, learning being faster the greater the discriminability of the elements. In the 1-29-0 pattern employed by Capaldi and Molina (1979), for example, the memory signaling 29 pellets, that is, that of 1 pellet, is very discriminably different from the memory signaling 0 pellets, that is, that of 29 pellets. But to learn the $20-10-0$ pattern, the rat must discriminate between the memory of 20 pellets, which signals 10 pellets, and the memory of 10 pellets, which signals 0 pellets, presumably a more difficult discrimination.

According to Hulse (1980), much, if not all, of the data reported by Capaldi and his associates is irrelevant to the rule-learning hypothesis because Capaldi employed experimental conditions different from those employed by Hulse and unfavorable to rule learning. Capaldi (e.g., Capaldi, Verry, \& Davidson, 1980b), on the other hand, has argued that memory variables have been extensively confounded in Hulse's experiments, the confounding being such that it appears that rats are learning rules when, for example, monotonic series are learned faster than nonmonotonic ones. We report two experiments in the present paper that employed procedures similar to those used in Hulse's laboratory, conditions thought to be favorable to rule learning (Hulse, 1980) and serial patterns that, like those employed by Capaldi and Molina (1979) and Capaldi et al. (1980a), yield one set of predictions from Capaldi's model and another from Hulse's model.

Experiment 1 was concerned with rats' rate of learning to anticipate the terminal 0-pellet element of an SM series or one of two WM series. According to Hulse (1978, p. 326), comparisons between SM and WM patterns provide a rigorous test of the hypothesis that patterns with simpler rules are easier to learn. Experiment 2 
investigated rats' ability to extrapolate either an SM or one of two WM patterns to include an additional element, behavior not previously addressed by the memory-discrimination model but reported by Fountain and Hulse (1981) to be better following initial training on an SM pattern than following initial training on NM or WM patterns.

\section{EXPERIMENT 1}

Hulse and Dorsky (1977) employed four daily pattern repetitions, or trials, with a $10-15$-sec interrun interval (IRI) separating runs to successive pattern elements and a 15-min interval between pattern repetitions, the intertrial interval (ITI). We also employed a 10-15-sec IRI, but our subjects received only two pattern repetitions each day, separated by a 15 -min ITI. Under some conditions, serial learning is facilitated by a short IRI, but the available evidence suggests that neither a short IRI nor four daily pattern repetitions are necessary conditions for rule learning (Haggbloom \& Ekdahl, 1985; Roitblat, Pologe, \& Scopatz, 1983).

Group SM received the series 14-7-3-1-0 and Group 5-5 received the series 14-5-5-1-0. As already noted, Hulse and Dorsky (1977) reported faster anticipation learning with the SM 14-7-3-1-0 series than with the WM 14-5-51-0 series, a finding consistent with the rule-learning model. The critical group in the present experiment is Group 1-1, given the WM series 14-9-1-1-0. Hulse's model predicts a slower rate of learning to anticipate 0 pellets in Group 1-1 than in Group SM, because the rule characterizing this WM pattern, that is, $\mathrm{E}(\mathrm{i})>\mathrm{E}(\mathrm{i}+1)$ and $E(3)=E(4)$, is more difficult to induce than that characterizing the SM pattern. Moreover, Hulse's model does not provide a basis for predicting a difference in rate of anticipation learning between Group 5-5 and Group 1-1, and would appear to expect none. Both patterns contain three instances of the less-than rule and one instance of an equals rule, the variables identified by Hulse as being of primary importance in serial learning.

Roitblat (1982) suggested that predictions about serial learning from the memory-discrimination model depend on assumptions about the nature of the psychological scale associated with the physical scale of food quantity, as this determines element discriminability. We concur with Roitblat's (1982) use of a logarithmic function, originally suggested by Capaldi (1967), to map physical quantities onto a psychological continuum. However, predictions from the memory-discrimination model depend far less on specific scaling assumptions than they do on recognizing that the model regards serial learning as a discrimination learning problem in which elements that predict large quantities function as positive discriminative stimuli and exercise excitatory stimulus control, and elements that predict small quantities function as negative discriminative cues and exercise inhibitory stimulus control. That substantial inhibition will accrue to memories of elements that predict small quantities is an important aspect of the model that has been overlooked by some (e.g., Roitblat, 1982).
The memory discrimination model predicts that learning to anticipate 0 pellets will be faster in Group 1-1, given the series 14-9-1-1-0, than in Group SM, given the series 14-7-3-1-0. In Group 1-1, the 1-pellet memory signaling the terminal 0-pellet element is very discriminably different from the only memory, that of the initial 14-pellet element, which signals a substantial reinforcement. The 9-pellet memory is also very discriminably different from the 1-pellet memory and, like the 1-pellet memory, is only very weakly reinforced. The discrimination problem for Group SM is predicted to be more difficult, because the elements of the 14-7-3-1-0 series are more evenly distributed (under virtually any scaling assumption) along the stimulus continuum, and are thus less discriminably different from one another, than the elements of the 14-91-1-0 series. Of at least equal importance, however, is the fact that the 1-pellet memory would be expected to acquire more inhibition in Group 1-1 than in Group SM. This is so because for Group 1-1, 1 pellet predicts a very small quantity, either 1 or 0 pellets, on two occasions per series presentation, whereas for Group SM, 1 pellet predicts 0 pellets on only a single occasion.

The memory-discrimination model also predicts that Group 5-5 will learn more slowly than Group SM, as reported by Hulse and Dorsky (1977). Here, the memory of 1 pellet, which signals the 0-pellet element, might receive more generalized associative strength from the more similar and quite strongly reinforced 5-pellet memory of the WM series than from the less similar and less strongly reinforced 7-pellet memory, or more similar but very weakly reinforced 3-pellet memory, of the SM series. Moreover, inhibition would be directly conditioned to the 1-pellet memory in both conditions, but that memory would receive more generalized inhibition from the 3-pellet memory of the SM series than from the 5-pellet memory of the 5-5 series.

\section{Method}

Subjects. The subjects were 12 male albino rats, approximately 90 days old at the beginning of the experiment, bred in the laboratory from Holtzman stock.

Apparatus. Rats were tested in a straight enclosed runway modeled after that used by Hulse and Dorsky (1977). The runway was $92 \mathrm{~cm}$ long, and a $30-\mathrm{cm}$ startbox and a $30-\mathrm{cm}$ goal area were separated from the rest of the runway by manually operated guillotine doors. The goal area contained an $11-\mathrm{cm}$ niche at a right angle to the runway and an unpainted goal cup positioned against the end wall of the goal area. The goal cup was a small block of wood with a 6-cm-diam hole drilled into it. The inside width of the runway was $10 \mathrm{~cm}$ and the inside height was $11 \mathrm{~cm}$. The nunway was painted flat black throughout. The start and goal areas were covered with hinged Plexiglas, and the runway proper was covered with hardware cloth. Raising the guillotine door between the startbox and the runway triggered a .01-sec timer, which was stopped when the rat entered the goalbox and interrupted a photobeam $20 \mathrm{~cm}$ into the goal area. Food reinforcement consisted of an appropriate number of .045-g Noyes pellets.

Procedure. Fourteen days prior to training, the rats were placed on a food-deprivation schedule, consisting of $12 \mathrm{~g}$ of food per day, which was maintained throughout the experiment. The amount of food received in the goalbox was subtracted from the daily ration. Water was always available. 
On Days 10-12 of deprivation, the rats were tamed by being handled in squads of two for 2 min per squad. On Day 13 of deprivation, the rats were placed individually in the runway with both guillotine doors open, and allowed to explore the runway for $5 \mathrm{~min}$ and obtain five food pellets, one located in the food cup and four scattered at random. This procedure was repeated once, with the rat being returned to a holding cage for $10-15$ min between exploration periods. On Day 14 of deprivation, the rats were placed individually in the startbox and released when the guillotine door was raised. During a $10-\mathrm{min}$ interval, they were allowed to explore the runway and obtain a single food pellet located in the food cup.

On Day 15 of deprivation, four rats were assigned randomly to each of three groups and experimental training was begun. On each day of training, eack rat received two trials, each trial consisting of five nuns separated by a 10-15-sec IRI, on one of three series of different numbers of $.045 \mathrm{-g}$ food pellets. Group SM received the series 14-7-3-1.0, Group 5-5 received the series 14-5-5-1-0, and Group 1-1 received the series 14-9-1-1-0. Rats were removed from the goalbox imunediately after consuming the reinforcement, or after $10 \mathrm{sec}$ in the case of nonreinforcement.

The subjects were run in squads of three, with one rat from each group. The internun interval was $10-15 \mathrm{sec}$, and the intertrial interval was $15 \mathrm{~min}$. If a rat failed to complete a run within $60 \mathrm{sec}$, it was placed in the goalbox. Training continued for 14 days.

\section{Results}

Running times on all runs on Days 1 and 2 were very long compared with those on subsequent days and, except for Group 1-1 on Day 2, showed no evidence of anticipation. Running times on each run collapsed across the next 6 days of training are shown for each group in the left panel of Figure 1. Comparable data for the last 6 days of training are shown in the right panel. As can be seen in Figure 1, only Groups SM and 1-1 showed evidence of anticipation of the 0 -pellet element, anticipation being better in Group 1-1 than in Group SM early in training (left panel) and nearly identical for those groups late in training (right panel).

Identical groups $x$ runs $x$ trials $x$ days analyses of variance were applied to the data shown in the left and right panels of Figure 1, and both analyses yielded a reliable groups $\times$ runs interaction $[\mathrm{Fs}(8,36)=2.33$ and 2.26, respectively, ps < .05]. Comparisons between running times on Run 1 versus Run 5 on each day showed that Group 1-1 ran reliably more slowly to 0 pellets (mean $=14.19 \mathrm{sec}$ ) than to 14 pellets $($ mean $=5.54 \mathrm{sec}$ ) by the 3rd day of training $[F(1,36)=11.86, p<.01]$ and on each day thereafter. Group SM, on the other hand, did not run reliably more slowly to the 0 -pellet element $($ mean $=8.84 \mathrm{sec})$ than to the 14-pellet element (mean $=$ $1.79 \mathrm{sec})$ until the 6th day of training $[\mathrm{F}(1,36)=7.88$, $\mathrm{p}<.01$ ] . Comparisons between groups on Run 5 for the data shown in the left panel of Figure 1 showed that Group 1-1 ran more slowly to the 0-pellet element than did Group SM $[F(1,25)=4.29, \mathrm{p}<.05]$ but that Group SM did not run more slowly than Group 5-5 $[F(1,25)=$ 3.871. ${ }^{1}$

Comparisons between times on Run 1 versus Run 5 for each group for the data shown in the right panel of Figure 1 showed that Groups SM and 1-1 ran more slowly to 0 pellets than to 14 pellets $[\mathrm{Fs}(1,36)=27.36$ and 22.71 , respectively, ps $<.01$, but that Group 5-5 did not $(\mathrm{F}<1)$. Comparisons between groups on Run 5

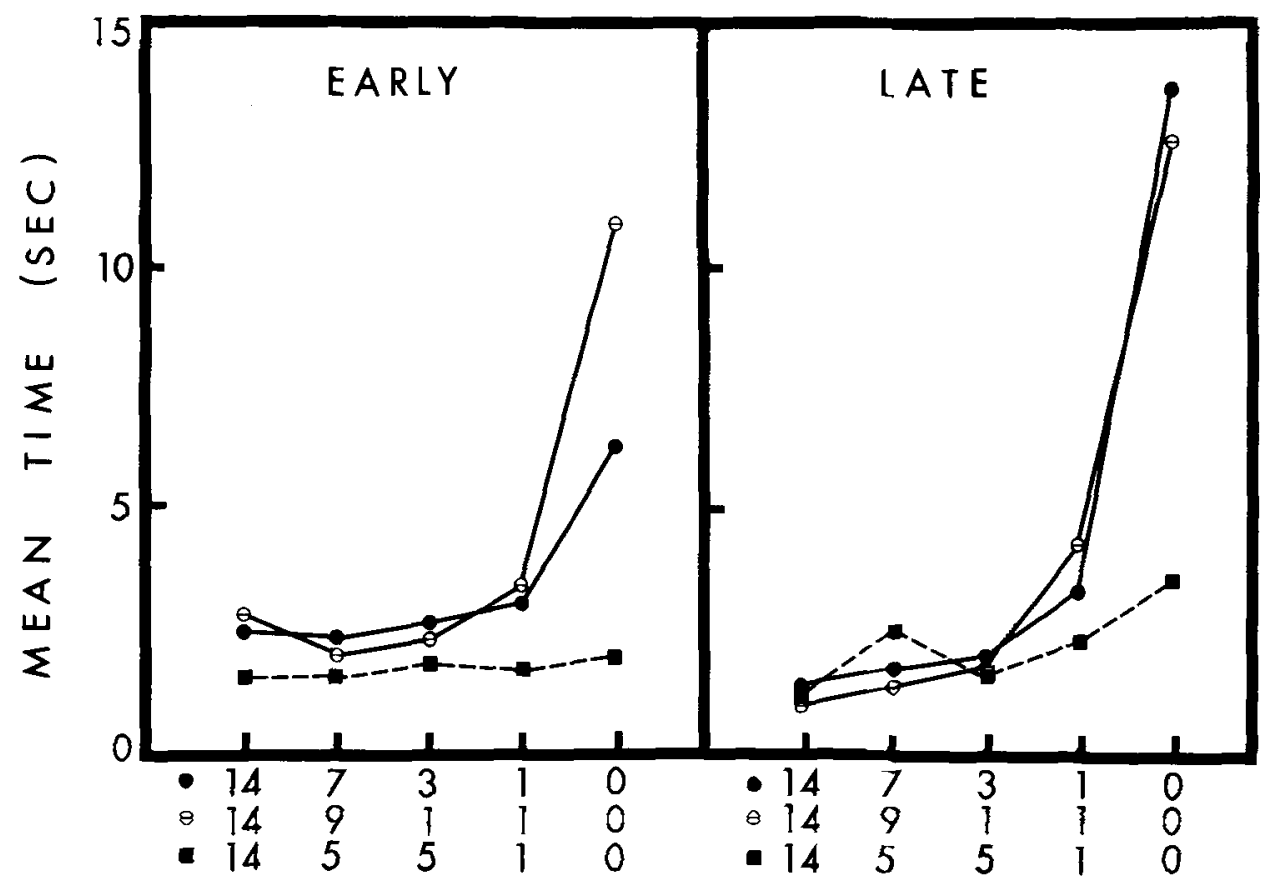

\section{PATTERN ELEMENTS}

Figure 1. Running times for each group in Experiment 1 on each run collapsed across blocks of 6 days early in training (left panel) and late in training (right panel). 
showed that Groups SM and 1-1 both ran more slowly than Group 5-5 [Fs $(1,40)=15.04$ and 6.91, ps $<.01$ and .05 , respectively], but those two groups did not differ from each other $(F<1)$ (see Note 1$)$.

\section{Discussion}

The finding here that anticipation of a terminal 0-pellet element occurred faster in a WM series consisting of highly discriminable elements (Group 1-1) than in an SM series or a WM series consisting of less discriminable elements (Group 5-5) is entirely consistent with the memory-discrimination model proposed by Capaldi and strongly inconsistent with the rule-learning model favored by Hulse. These results suggest that previous, similar findings obtained with a relatively long IRI (Capaldi \& Molina, 1979) or relatively short series (Capaldi et al., 1980a) were probably not a consequence of those conditions' being unfavorable to rule learning, as suggested by Hulse (1980), but rather, as suggested by Capaldi and his associates, were a function of the signal value and discriminability of the elements employed in those series.

\section{EXPERIMENT 2}

Fountain and Hulse (1981) proposed that an advantage of the rule-learning model is that predictions about animal behavior can be generated from it that would not be forthcoming from other approaches. For example, acquisition of a rule would imply the ability to extrapolate or extend a series. To investigate this possibility, they trained rats for 13 days on either the SM pattern 14-7-3-1, the WM pattern 14-5-5-1, or the NM pattern 14-3-7-1. On Day 14, the test (extrapolation) phase of the experiment began and all rats experienced the addition of a 0 -pellet element to the end of their respective patterns. The results of the initial trial (first pattern repetition) containing the added element indicated that rats in the SM condition anticipated, and ran slowly to, the reduced quantity before actually experiencing it. The rats in the WM and NM conditions ran fast to the added element, showing no sign of anticipation.

The purpose of the present experiment was to investigate whether the memory-discrimination model could predict extrapolation behavior as a function of element discriminability that would not be predicted by the rulelearning model. To accomplish this, rats were trained in Phase 1 either on an SM (14-7-3-1) series (Group SM) or on one of two WM series (14-5-5-1, Group 5-5, or 14-9-1-1, Group 1-1), and then, in the test phase, given the additional 0-pellet element at the end of their respective patterns.

In what follows, the term "extrapolation" is used to refer to the behavior of running more slowly to a 0-pellet element added to the end of a learned series than to other pattern elements without the attendant assumption that that behavior is produced by extension of a rule. According to the memory-discrimination model, addition of a 0-pellet element to test for extrapolation would produce the best anticipation of 0 pellets in Group 1-1. This is so because the training phase would have established the memory of a 1-pellet reward as a negative discriminative stimulus signaling a very small, 1-pellet reward. The worst extrapolation would be predicted for Group 5-5. In that group, the memory of 5 pellets signals both 5 pellets (Run 3 ) and 1 pellet (Run 4) and responding following 1 pellet would depend on generalized tendencies from other elements. Most of the generalization would be supplied by the ambiguous 5-pellet element which is the element in the 14-5-5-1 series most similar to 1 pellet. An intermediate level of extrapolation would be predicted for Group SM where, during training, the memory of 3 pellets would be established as a signal for 1 pellet and inhibitory response tendencies could generalize from that element to the 1-pellet element. The rule-learning model, on the other hand, would predict the best extrapolation in Group SM, given a structurally simple pattern. The equally complex 14-5-5-1 and 14-9-1-1 patterns experienced by Groups 5-5 and 1-1 would be expected to produce comparably inferior extrapolation.

\section{Method}

Subjects. The subjects were 24 male albino rats, $90-110$ days old at the beginning of training, bred in the laboratory from Holtzman stock.

Apparatus. The apparatus was the same as in Experiment 1.

Procedure. The experiment was conducted in two identical replications with three rats per group in each replication. All procedures prior to the 1st day of training were the same as in Experiment 1. On Day 1 of training, each rat received two trials, a trial consisting of four successive runs to a series of different numbers of $.045-\mathrm{g}$ food pellets. On Days 2-13, each rat received four trials per day. The IRI was 10-15 sec and the ITI was $15 \mathrm{~min}$. Group SM received the series 14-7-3-1, Group 5-5 received the series 14-5-5-1, and Group 1-1 received the series 14-9-1-1. The initial training on the four-element patterns was followed by 3 days of extrapolation tests with the added 0-pellet element. As in the Fountain and Hulse (1981) experiment, rats received the same four-element pattern experienced in the training phase on Trial 1 of the first test day. On all remaining trials, each group experienced the 0-pellet element in the fifth position in the series. All trial administration procedures were the same as in Experiment 1.

\section{Results}

Test trials. Behavior on the first presentation of the test patterns containing the added 0-pellet element provides the best indication of each group's ability to correctly extrapolate the pattern strictly as a function of initial learning. Thus, as in the Fountain and Hulse (1981) experiment, the data from the first test pattern presentation are emphasized here. Behavior on subsequent presentations of the test series, however, was essentially the same as on the first presentation.

Figure 2 displays the mean running times for each group on each run of the first extrapolation test trial. As can be seen in Figure 2, Groups 1-1 and SM correctly anticipated presentation of the 0-pellet element, as indicated by slower running times on that run than on earlier runs in the series. Extrapolation was somewhat better in Group 1-1 than in Group SM. Group 5-5, on the other hand, 


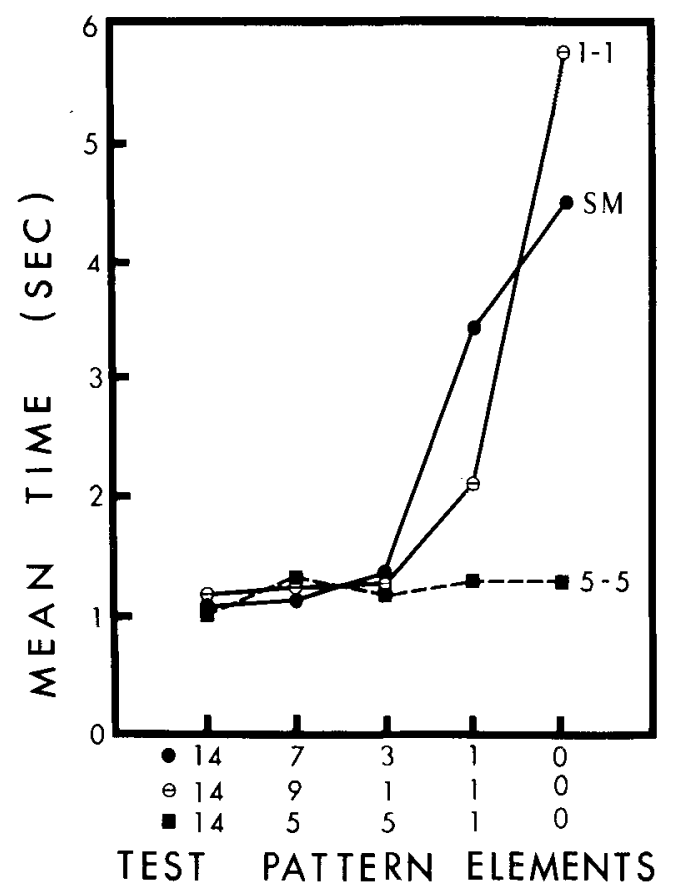

Figure 2. Running times for each group in Experiment 2 on each run of the first extrapolation test trial.

showed no sign of anticipation, as evidenced by comparably fast running times to all five elements in that group.

A groups $\times$ replications $\times$ runs analysis of variance was performed on the data shown in Figure 2. The analysis showed no differences between replications $[F(1,12)$ $=2.10$ ] and no interactions with replications [largest $F(8,48)=1.65$ for the groups $\times$ replications $\times$ runs interaction]. The better anticipation of the 1- and 0-pellet elements in Groups 1-1 and SM than in Group 5-5 was reflected in the reliable groups $\times$ runs interaction $[F(8,48)=2.34, p<.05]$. Comparisons between running times on Run 5 versus Run 1 showed that Group SM ran more slowly to 0 pellets than to 14 pellets $[F(1,48)=$ $11.58, \mathrm{p}<.01]$, as did Group $1-1[\mathrm{~F}(1,48)=21.70$, $\mathrm{p}<.01]$. Group 5-5 did not run more slowly on Run 5 than on Run 1 ( $F<1)$. Additional comparisons showed that Groups 1-1 and SM ran more slowly on Run 5 than did Group 5-5 [Fs $(1,12)=10.39$ and 10.52 , respectively, ps $<.01]$ but did not differ from each other $[F(1,12)=$ 1.60].

Fountain and Hulse (1981) reported analyses of all seven test trials they administered. An analysis of the first seven test trials here yielded a significant main effect of runs $[F(4,48)=14.31, p<.01]$ and a significant groups $\times$ runs interaction $[F(8,48)=2.23, p<.05]$. Collapsing across all seven trials, mean running time was faster in Group SM on Run 1 (mean $=1.22$ ) than on Run 5 $($ mean $=5.09)[F(1,48)=29.82, p<.01]$, and was similarly faster in Group $1-1$ on Run 1 (mean $=1.23$ ) than on Run 5 (mean $=4.26)[F(1,48)=18.26$, p < $.01]$. Group 5-5, on the other hand, did not respond differentially on Runs $1($ mean $=1.19)$ and $5($ mean $=2.13)$
$[\mathrm{F}(1,48)=1.77]$. Groups SM and 1-1 did not differ from each other in Run 5 times $[\mathrm{F}(1,48)=1.28]$, but both of those groups ran more slowly than Group 5-5 [Fs $(1,48)=$ 16.45 and 8.54 , respectively, ps $<.01$ ].

A control analysis was performed by Fountain and Hulse (1981) to compare behavior on the first pattern repetition of Day 14 and Runs 1 to 4 of the second pattern repetition of Day 14, the first trial with a 0-pellet element added to the series. Because subjects did not experience the 0-pellet element until Run 5 of the second trial on Day 14, behavior on Runs 1-4 of Trial 2 should be essentially the same as behavior on Trial 1 . As in the Fountain and Hulse experiment, this analysis yielded a reliable runs effect $[F(3,36)=7.10, p<.01]$ but no significant effect for groups or interactions with groups (ps $>.05$ ).

Initial training. Table 1 presents running times for each group on each run averaged over the last 6 days of initial training. As can be seen, running times increased over runs in each group, the increase being most substantial in Group 1-1, smallest in Group 5-5, and intermediate in Group SM. Analysis of variance applied to the data summarized in Table 1 yielded a reliable groups $\times$ runs interaction $[F(6,36)=3.45, p<.01]$. Comparisons between running times on Run 4 versus Run 1 were reliable for Group SM $[F(1,36)=17.36, p<.01]$ and for Group $1-1[F(1,36)=47.06, p<.01]$, but not for Group 5-5 $[F(1,36)=2.18]$. Comparisons between groups on Run 4 showed that Group 1-1 ran more slowly to the 1-pellet element than did Group $S M[F(1,12)=7.56, p<.05]$ and that Group SM, in turn, ran more slowly than Group $5-5[F(1,12)=7.16, p<.05]$.

\section{Discussion}

In Phase 1, Group 1-1, trained on the complex WM pattern 14-9-1-1, consisting of highly discriminable elements, showed better anticipation of the terminal 1-pellet element than did Group SM, trained on the relatively simple pattern 14-7-3-1, consisting of less discriminable elements. Group 1-1 also anticipated the terminal 1-pellet element better than Group 5-5, trained on the equally complex 14-5-5-1 pattern, a pattern in which the memory signaling the terminal 1-pellet element was ambiguous. These results exactly parallel the results of Experiment 1, obtained with similar, but longer, series.

In the test phase, Group 1-1 did not, as predicted, extrapolate the series significantly better than Group SM, but the difference was in that direction and definitely not in the direction predicted by the rule-learning model. The

Table 1

Running Times on Each Run Averaged Over the Last 6 Days of Phase 1 in Experiment 2

\begin{tabular}{lcccc}
\hline & \multicolumn{4}{c}{ Run } \\
\cline { 2 - 5 } Group & 1 & 2 & 3 & 4 \\
\hline SM & 1.22 & 1.31 & 1.64 & 2.73 \\
$1-1$ & 1.25 & 1.30 & 1.64 & 3.74 \\
$5-5$ & 1.21 & 1.25 & 1.44 & 1.75 \\
\hline
\end{tabular}


better extrapolation in Group SM than in Group 5-5 is consistent with the rule-learning model and with results reported by Fountain and Hulse (1981), but can also be predicted by the memory-discrimination model. However, the fact that extrapolation was better in Group 1-1 than in Group 5-5 is not in agreement with the rule-learning model, because both groups received equally complex WM patterns. The superior extrapolation in Group 1-1 over Group 5-5 was predicted by the memory-discrimination model. The pattern of results obtained here in both the training and test phases suggests that the extrapolation results obtained by Fountain and Hulse (1981) might have been a consequence of the signal value and discriminability of elements rather than the differential acquisition of rules.

\section{GENERAL DISCUSSION}

The two experiments reported here employed procedures very similar to those employed in Hulse's laboratory and serial patterns for which the rule-learning and memory-discrimination models made different predictions. Thus, the pattern of results obtained here, which is in substantial agreement with the memory-discrimination model, would not appear to be attributable to the use of conditions unfavorable to rule learning.

It is important to recognize that, in the absence of a group trained like Group 1-1 in either of the present experiments, the better performance of Group SM over Group 5-5 could readily be attributed, as it was for those conditions in Hulse and Dorsky (1977) and Fountain and Hulse (1981), to easier acquisition of a rule describing the relatively simple SM pattern than of the more complex WM pattern experienced by Group 5-5. However, given the superior anticipation and extrapolation behavior of Group 1-1 on an equally complex WM pattern, there is no basis for attributing the behavioral difference between Groups SM and 5-5 to the putative relationship between pattern complexity and rule learning. Rather, the entire pattern of results obtained here can be explained in terms of the signal value and discriminability of pattern elements. Of course, if the behavior of Groups SM and 5-5 in the present experiments was not due to differential rule learning, it seems unlikely that the behavior of their counterparts in Hulse and Dorsky (1977) and Fountain and Hulse (1981), obtained under very similar conditions, was regulated by rule learning.
The present results complement results reported by Capaldi, Nawrocki, Miller, and Verry (1985) showing that, even under conditions thought to be optimal for rule learning, complex patterns may be learned more rapidly than simple patterns. The present results also show that the memory-discrimination model can successfully predict extrapolation, behavior not previously addressed by that model.

\section{REFERENCES}

CAPALDI, E. J. (1967). A sequential hypothesis of instrumental learning. In K. W. Spence \& J. T. Spence (Eds.), The psychology of learning and motivation (Vol. 1, pp. 67-156). New York: Academic Press.

Capaldi, E. J., \& Molina, P. (1979). Element discriminability as a determinant of serial pattern learning. Animal Learning \& Behavior, 7, 318-322.

Capaldi, E. J., Nawrocki, T. M., Miller, D. J., \& Verry, D. R. (1985). An examination into some variables said to affect serial learning. Animal Leaming \& Behavior, 13, 129-136.

Capaldi, E. J., Verry, D. R., \& Davidson, T. L. (1980a). Memory, serial anticipation pattern learning, and transfer in rats. Animal Learning \& Behavior, 8, 575-585.

Capaldi, E. J., Verry, D. R., \& Davidson, T. L. (1980b). Why rule encoding by animals in serial learning remains to be established. Animal Learning \& Behavior, 8, 691-692.

Fountain, S. B., \& Hulse, S. H. (1981). Extrapolation of serial stimulus patterns by rats. Animal Learning \& Behavior, 9, 381-384.

HaGgBloOM, S. J., EKDAHL, M. W. (1985). Effects of interrun interval on serial learning. Animal Learning \& Behavior, 13, 98-102.

HULSE, S. H., (1978). Cognitive structure and serial pattern learning by rats. In S. H. Hulse, H. Fowler, \& W. K. Honig (Eds.), Cognitive processes in animal behavior. Hillsdale, $\mathrm{NJ}$ : Erlbaum.

Hulse, S. H. (1980). The case of the missing rule: Memory for reward vs. formal structure in serial-pattern learning by rats. Animal Learning \& Behavior, 8, 689-690.

Hulse, S. H., Dorsky, N. P. (1977). Structural complexity as a determinant of serial pattern learning. Learning \& Motivation, 8, 488-506.

RoItblat, H. L. (1982). The meaning of representation in animal memory. Behavioral \& Brain Sciences, 5, 533-406.

Roitblat, H. L., Pologe, B., Scopatz, R. A. (1983). The representation of items in serial position. Animal Learning \& Behavior, 11, 489-498.

WINER, B. J. (1971). Statistical principles in experimental design (2nd ed.). New York: McGraw-Hill.

\section{NOTE}

1. This test required a pooled error term. The degrees of freedom were adjusted employing the Satterthwaite method recommended by Winer (1971).

(Manuscript received February 4, 1985; revision accepted for publication July 22, 1985.) 